\title{
Optimal Shape of a Gamma-ray Collimator: single vs double knife edge
}

\author{
Albert Metz $^{1^{*}}$, and Alfred Hogenbirk ${ }^{2}$ \\ ${ }^{1}$ NRG, Consultancy \& Services, 6800 ES Arnhem, The Netherlands \\ ${ }^{2}$ NRG, Nuclear Operations, $1755 Z$ Z Petten, The Netherlands
}

\begin{abstract}
Gamma-ray collimators in nuclear waste scanners are used for selecting a narrow vertical segment in activity measurements of waste vessels. The system that is used by NRG uses tapered slit collimators of both the single and double knife edge type. The properties of these collimators were investigated by means of Monte Carlo simulations. We found that single knife edge collimators are highly preferable for a conservative estimate of the activity of the waste vessels. These collimators show much less dependence on the angle of incidence of the radiation than double knife edge collimators. This conclusion also applies to cylindrical collimators of the single knife edge type, that are generally used in medical imaging spectroscopy.
\end{abstract}

\section{Introduction}

For the characterization of radioactive waste by means of $\gamma$-radiation two types of scanners are available. The Tomographic Gamma Scanner (TGS) provides a two dimensional image of slabs from a waste vessel, with a typical height of a few centimetres [1]. The Segmented Gamma Scanner (SGS) also makes use of measurements from different vertical segments, but can only determine the overall activity of a segment [2]. In both types of scanners the waste vessel is usually rotated about the vertical axis as shown in Figure 1.

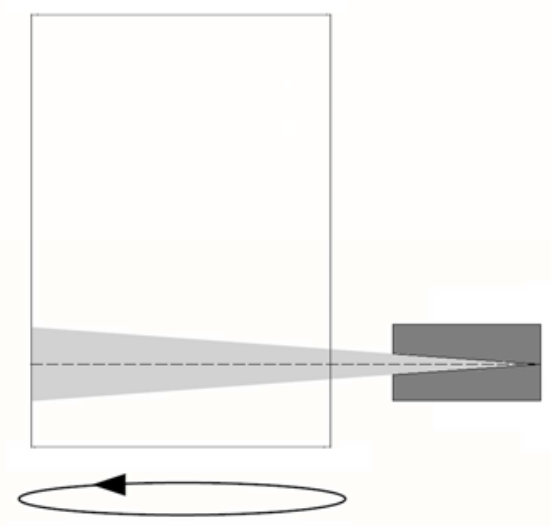

Fig. 1. Schematic view of a waste scanner. Radiation from a segment of the rotating waste vessel (left) is collected in a collimated beam.

In order to obtain a spatial resolution in the axial direction (i.e. to be able to differentiate between signals from different vertical segments), it is necessary to limit the angular acceptance of the detection system. This can be arranged by means of a collimating system, which ideally should provide a response that is independent of the angle of incidence within the measured axial segment. Because of the finite angle of acceptance, and also because the vessel is rotated, it is unavoidable that measurements from different segments have some overlap.

Conditions for collimation in the horizontal direction are less severe. When tomographic or imaging techniques can be applied, the horizontal location of the waste within the segment can be determined, so that an efficiency correction can be applied.

For high-level radioactive waste the SGS technique is usually applied, as is done in the VINISH setup at NRG [3]. In this setup the collimating system consists of separate horizontal and vertical collimators, each of which collimates only in one direction. These are mounted on a sliding system, so that collimators can be selected with different apertures. By reducing the collimator aperture, the count rate in the detector can be reduced, so that waste vessels with both high and low activities can be analysed. For the highest activities the collimator aperture should be reduced as much as possible. In that case a considerable fraction of the transmitted beam passes through the collimator body, which is usually made from tungsten.

In this paper we use Monte Carlo simulations to investigate the behaviour of collimating systems with different geometries. These geometries are described in section 2 of this paper. The setup of the simulations is described in section 3. Results on the effect of the acceptance angle, $\gamma$-energy, split width and collimator thickness are reported in section 4 . In section 5 results are reported for cylindrical collimators, that are

\footnotetext{
Corresponding author: metz@nrg.eu
} 
commonly used in medical imaging applications, and a comparison with published theoretical results is made. Discussion and conclusions are presented in sections 6 and 7.

\section{Collimator types}

In this paper we consider two collimator types that have an (apparently) well-defined angle of acceptance. One type has a so-called double knife edge (DKE) and is shown in Figure 2. The other is of the single knife edge type (SKE) and is shown in Figure 3. The angles $\alpha$ and $\phi$ denote, respectively, the acceptance angle of the collimator and the angle between the - supposedly parallel - beam and the surface normal.

Initially we will consider collimators that have a 1-dimensional slit, i.e. they are tapered in the plane of the drawings, but their cross sections do not change in the direction parallel to this plane.

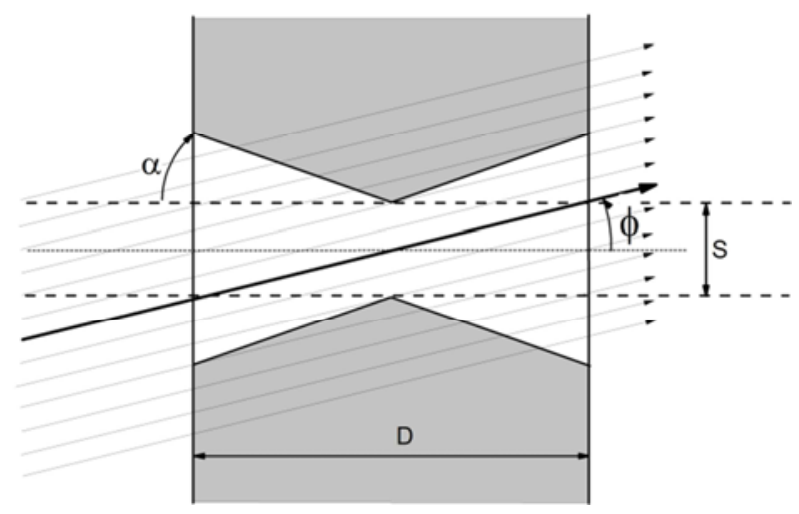

Fig. 2. Cross section of the double knife edge collimator. The symbol $\mathrm{S}$ denotes the collimator split, D is the thickness of the collimator. The other symbols are described in the text.

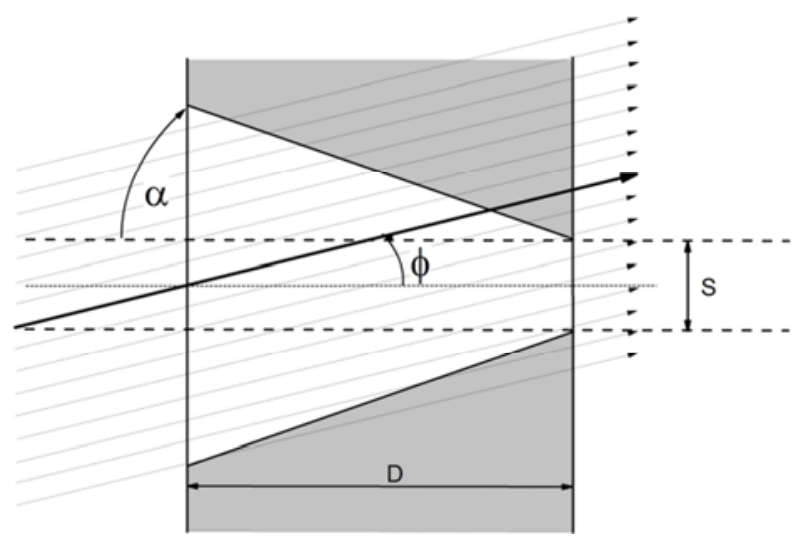

Fig. 3. Cross section of the single knife edge collimator. The meaning of the symbols is as in Fig.2.

\section{Monte Carlo Simulations}

The imaging properties of cylindrical collimators have been investigated by various authors. For some geometries analytical results have been obtained. These will be discussed in section 5 . For the 1D knife edge collimators that form the main subject of this paper no analytical results have been published to the authors' knowledge.

Therefore Monte Carlo simulations have been carried out with MCNPX [4]. For the 1D-cases a line source was used, producing a parallel beam of mono-energetic $\gamma$-rays that impinges on the left side of the collimator, as shown in Figure 4. The resulting parallel beam closely approximates the actual situation in a waste scanning setup, where the source detector distance is usually much larger than the opening of the collimator. Transmitted particles were tallied using a flux tally (F2) for a circular surface directly behind the collimators. This is a model for the gamma detector, which will be placed on this location in the case of an actual measurement. The length of the line source $(10 \mathrm{~cm})$ was chosen sufficiently large to ensure that both the collimator and the tally surface (diameter $5 \mathrm{~cm}$ ) are fully covered by the $\gamma$-beam for all relevant angles of incidence $\phi$. An energy filter of $\pm 5 \mathrm{keV}$ around the initial $\gamma$-energy was applied, so that only unscattered photons were selected. For these photons the transmission of the collimator was calculated by comparison with the photon flux for the case where the collimator is replaced by an air volume in an otherwise unchanged geometry. For the 1D-cases a total of $10^{6}$ particle histories was sufficient to obtain good statistics, with relative errors of the order of $1 \%$ for a transmission of $10^{-2}$, and $5 \%$ for a transmission of $10^{-3}$.

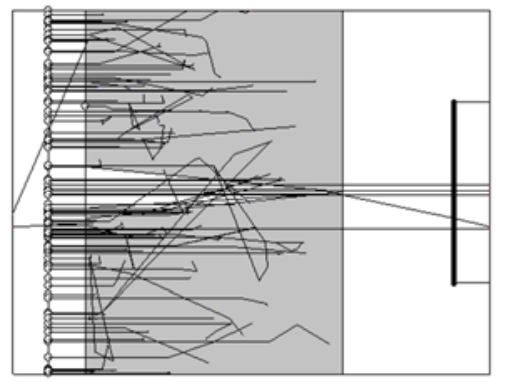

Fig. 4. Cross section of the MCNP model. A parallel beam of $\gamma$-rays emerges from the line source on the left. The thick vertical line on the right marks the tally plane. 


\section{Results for 1D-collimators}

In all our simulations we find that DKE collimators show a strong dependence of the transmission on the angle of incidence $\phi$, whereas SKE collimators have a nearly $\phi$-independent response within the angle of acceptance $\alpha$. As an example we show the results for a typical case, using a photon-energy of $1 \mathrm{MeV}$, a tungsten collimator with a thickness $D$ of $7 \mathrm{~cm}$, zero split $S$ and an acceptance angle $\alpha$ of $5.7^{\circ}$ (corresponding to $\tan (\alpha)=$ $0.1)$. The input parameters are summarized in Table 1 .

Table 1. MCNP Input parameters for 1D-collimators.

\begin{tabular}{|c|c|}
\hline Material & Tungsten \\
\hline$D(\mathrm{~cm})$ & 7.0 \\
\hline$S(\mathrm{~cm})$ & 0.0 \\
\hline $\tan (\alpha)$ & 0.1 \\
\hline $\mathrm{E}(\mathrm{MeV})$ & 1.0 \\
\hline $\mathrm{nps}$ & $10^{6}$ \\
\hline
\end{tabular}

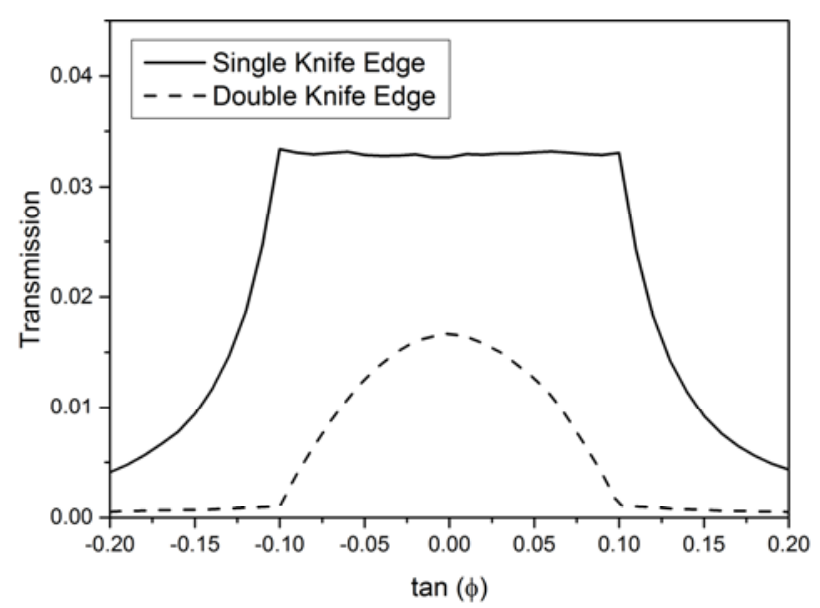

Fig. 5. Transmission curves for single and double knife edge collimators. The relevant parameters are given in Table 1.

For angles of incidence within the acceptance angle of the collimator, ranging from $\tan (\phi)=-0.1$ to $\tan (\phi)=$ 0.1 , the SKE transmission curve is practically independent of the angle of incidence $\phi$, but it shows considerable 'leakage' outside of this region. The DKE collimator, on the other hand, has a strong $\phi$-dependence inside the acceptance angle, but outside of this region it drops to zero much more rapidly.

\subsection{Effect of acceptance angle}

For practical applications it is of interest to investigate the dependence on the acceptance angle. Results for $\tan (\alpha)=0.01$ to 0.1 (with $\alpha$ ranging from $0.57^{\circ}$ to $5.7^{\circ}$ ) are shown in Figures 6 and 7.

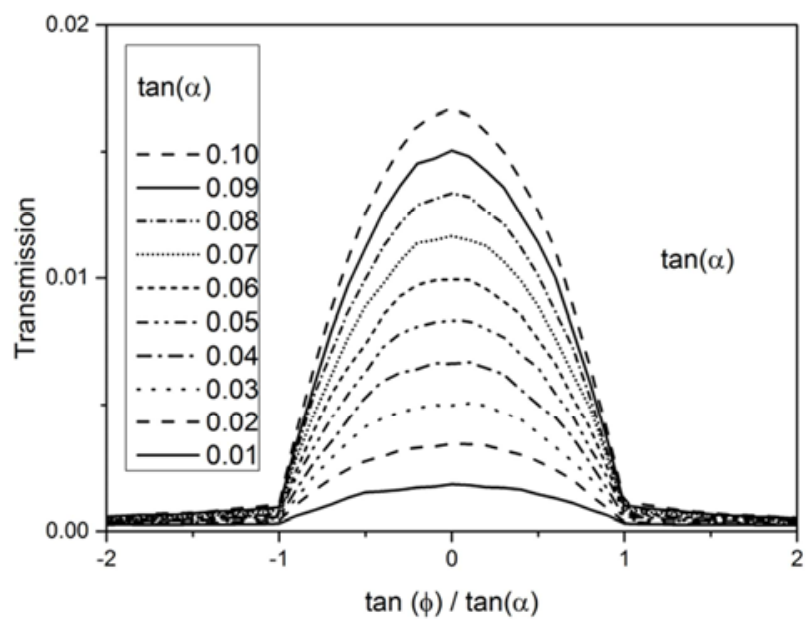

Fig. 6. Transmission curves for DKE collimators for various acceptance angles $\alpha$. The horizontal axis is scaled to $\tan \alpha$, to allow for comparison of the different cases. The relevant parameters are given in Table 1 .



Fig. 7. Transmission curves for SKE collimators for various acceptance angles $\alpha$.

From these curves it is clear that the qualitative behaviour of the two collimator types (flat response for SKE, versus strongly varying response for DKE) is independent of the acceptance angle $\alpha$. 


\subsection{Effect of $\gamma$-Energy}

A further point of interest is the dependence of the transmission upon the $\gamma$-energy. In Figures 8 and 9 transmission curves for various energies are shown, ranging from 0.10 to $1.50 \mathrm{MeV}$.

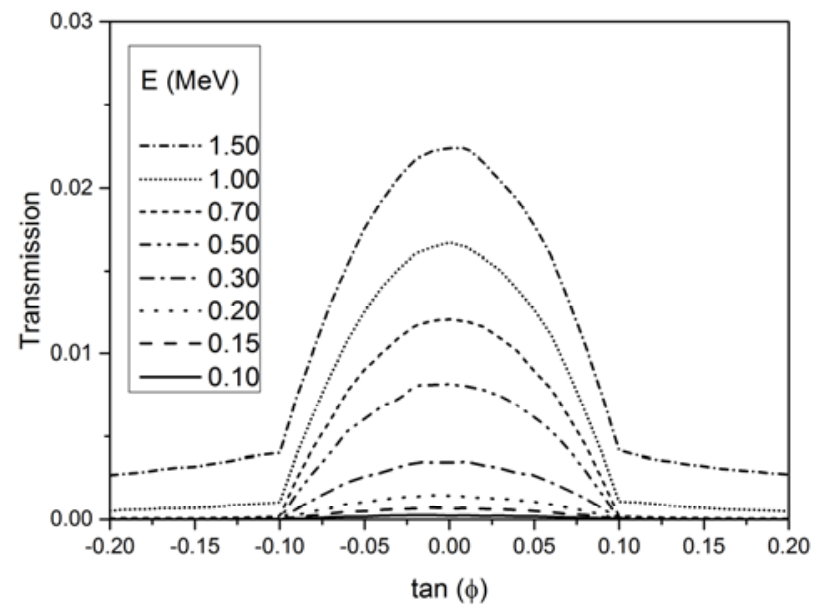

Fig. 8. Transmission curves for DKE collimators for various opening $\gamma$-energies $\mathrm{E}$. The relevant parameters are given in Table 1 .

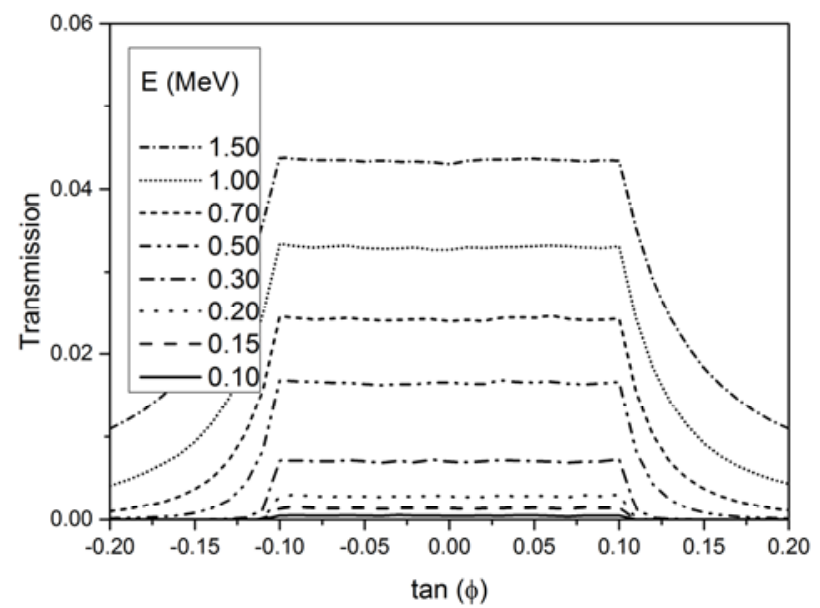

Fig. 9. Transmission curves for SKE collimators for various opening $\gamma$-energies E. The relevant parameters are given in Table 1.

From the transmission curves in Figure 9 it can be observed that the amount of leakage outside of the acceptance angle $\alpha$, which is typical for the SKE collimator, increases with $\gamma$-energy. Some leakage is also observed for the DKE collimator (Figure 10), but in this case the effect is much smaller.

\subsection{Effect of Collimator Split}

When a moderate reduction of the flux is desired, e.g. when measuring a waste vessel of medium or low activity, it is desirable to increase the transmission of the collimator system. This can in principle be achieved by using a collimator split $S$ (see Figures 2 and 3) different from zero. In Figures 10 and 11 transmission curves for various collimator splits are shown, ranging from 0.1 to $1.0 \mathrm{~cm}$.

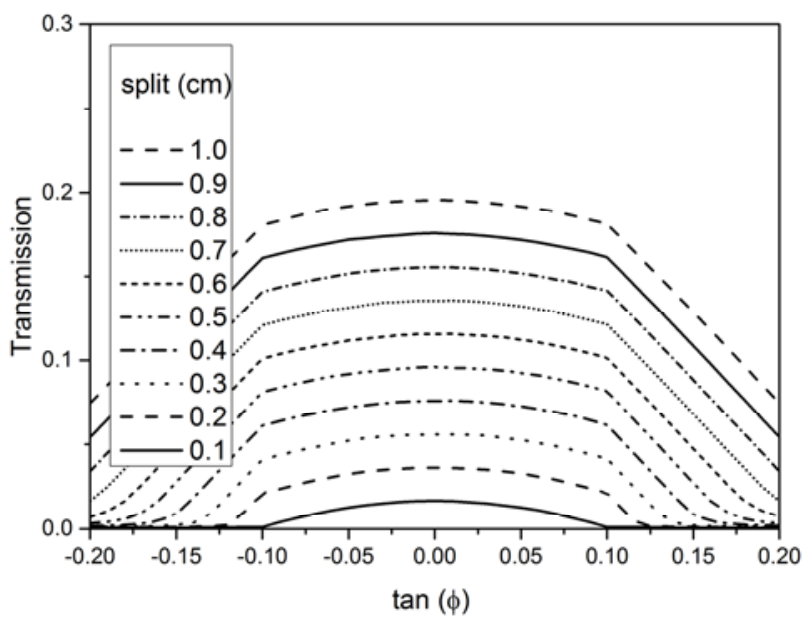

Fig. 10. Transmission curves for DKE collimators for various values of the collimator split width $S$. The other relevant parameters are given in Table 1.

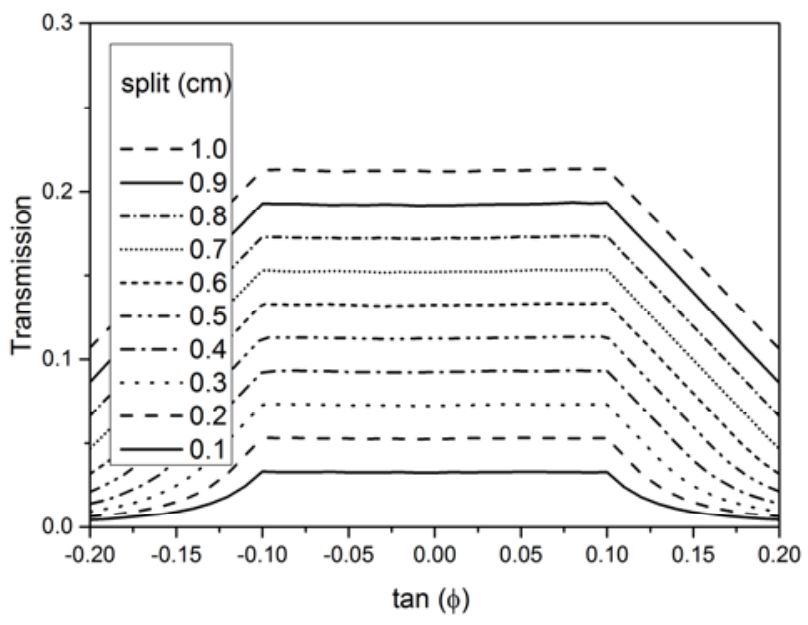

Fig. 11. Transmission curves for SKE collimators for various values of the collimator split width $S$. The other relevant parameters are given in Table 1.

From the transmission curves in Figures 10 and 11 it can be observed, that the transmission increases with increasing split width. The SKE curves (Figure 11) remain flat within the acceptance angle. The DKE curves (Figure 10) become relatively less curved with increasing split width. 


\subsection{Effect of Collimator Thickness}

In this section the dependence of the transmission upon the collimator thickness $D$ is studied. In particular it is interesting to investigate to what extent the leakage of $\gamma$ flux outside of the acceptance angle $\alpha$ can be reduced by increasing the collimator thickness. Within a practical thickness range from $D=3 \mathrm{~cm}$ up to $9 \mathrm{~cm}$ such effects can be well observed for a $\gamma$-energy of $0.5 \mathrm{MeV}$. As in the other simulations an acceptance angle with $\tan \alpha=0.1$ and zero split were used. The results are shown in Figures 12 and 13.

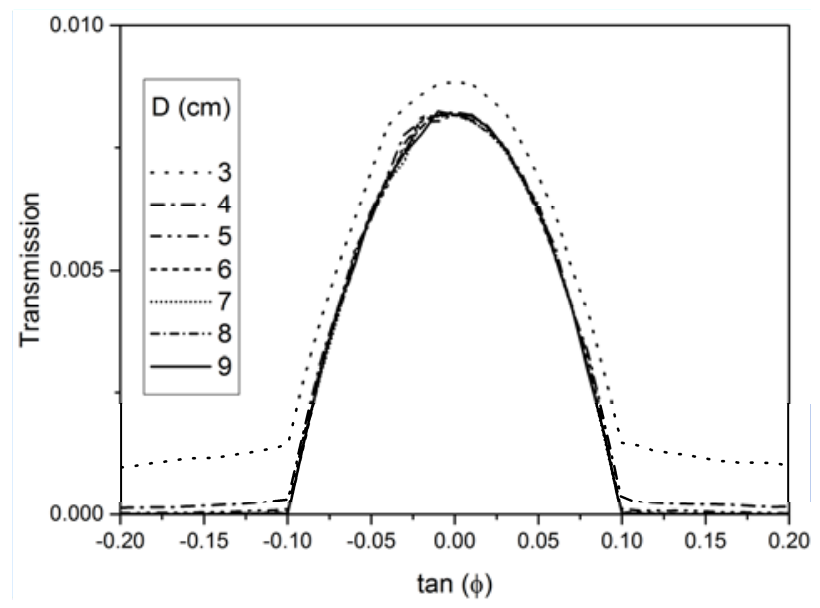

Fig. 12. Transmission curves for DKE collimators for various values of the collimator thickness $D$. The other relevant parameters are given in Table 1 with the exception of the $\gamma$ energy $E$ which is set to $0.5 \mathrm{MeV}$.

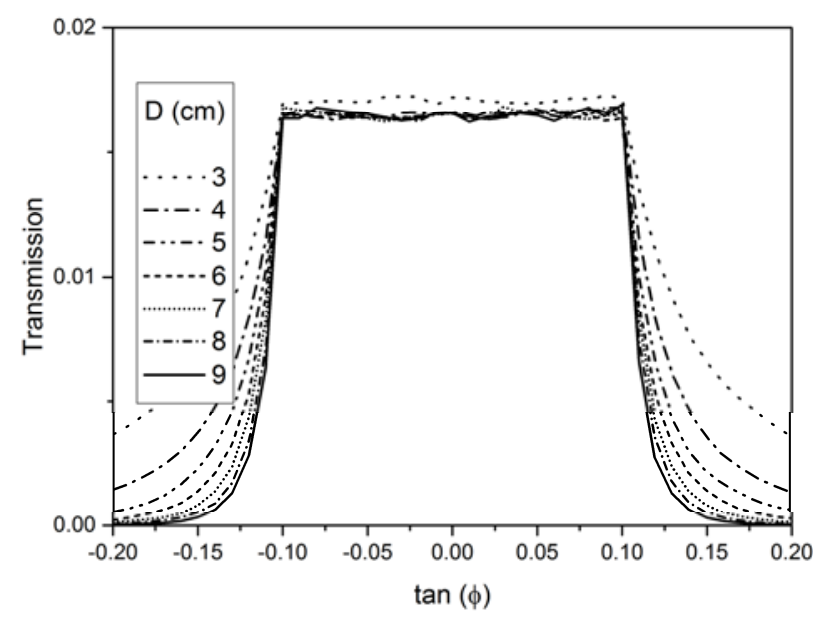

Fig. 13. Transmission curves for SKE collimators for various values of the collimator thickness $D$. The other relevant parameters are given in Table 1 with the exception of the $\gamma$ energy $E$ which is set to $0.5 \mathrm{MeV}$.

From the results in Figures 13 it can be observed that the transmission within the angle of acceptance is largely independent of the collimator thickness, but that the amount of leakage for the SKE collimator can be significantly reduced by increasing the collimator thickness. For the DKE collimator both the transmission and the leakage can be seen to be largely independent of the collimator thickness, with the exception of the case $D=3 \mathrm{~cm}$, which is insufficient to complete block $0.5 \mathrm{MeV} \gamma$-rays outside of the acceptance angle of the collimator.

\section{Results for cylindrical collimators}

Cylindrical collimators are widely used in (medical) imaging applications. In those applications the photon energy is lower and the collimator dimensions are smaller than those commonly used in waste scanners. Moreover the distance from the source to the collimator is chosen relatively small, to achieve a large magnification. In some cases researchers have even used an 'inverted' single knife edge collimator (which diverges from a narrow opening near the source to a wider opening near the detector), to make the distance to the source even smaller [5]. From a qualitative understanding of the physical principles - as discussed in section 6 - it seems likely that the differences that we have observed between one dimensional SKE and DKE collimators, will also occur for cylindrical collimators, with a $3 \mathrm{D}$ conical opening.

The behaviour of cylindrical collimators for imaging purposes has been extensively studied, with special attention for imaging properties, in particular the spatial resolution. In a number of publications the so called sensitivity has also been studied, a quantity that is equivalent to the transmission reported in this paper. Analytical results for sensitivity of the DKE collimator have been reported by Metzler et al. [6] and for the SKE case by Alnaghy et al. [7]. These authors make a distinction between the geometric sensitivity, which accounts for the photons that pass through the collimator aperture, and the penetration sensitivity, that accounts for the photons that pass through the collimator walls. By choosing a split of zero width, the geometric sensitivity can be reduced to zero, so that the transmission is completely described by the penetration sensitivity $S_{\text {pen. }}$.

For the cylindrical DKE collimator with zero split (No-Hole Approximation) Metzler et al. [6] present a formula, which can be rewritten as:

$S_{\text {pen;DKE }}(\phi) \approx C \times \cos ^{5} \phi \times\left(\tan ^{2} \alpha-\tan ^{2} \phi\right)^{3 / 2}$

where $C$ is a constant, our $\phi$ corresponds to $\pi / 2-\theta$ and our $\alpha$ to $\alpha / 2$ in [6].

The formula that Alnaghy et al. [7] present for the cylindrical SKE can be rewritten as:

$S_{\mathrm{pen} ; \mathrm{SKE}}(\phi) \approx C \times \cos ^{5} \phi \times\left(1-\frac{\cos ^{2} \alpha}{\cos ^{2} \phi}\right)^{\frac{3}{2}}$

where $C$ is a constant, our $\phi$ corresponds to $\pi / 2-\theta$ and our $\alpha$ to $\alpha / 2$ in [7]. 


\subsection{Effect of $\gamma$-Energy for cylindrical collimators}

We have investigated the transmission curves for cylindrical collimators for various energies, with the parameters given in Table 2. Because the transmission of the cylindrical collimator is much lower, a higher number of particle histories $\left(\mathrm{nps}=10^{8}\right.$ ) was required to achieve sufficient statistics, with relative errors of the order of $0.3 \%$ for a transmission of $10^{-3}$, and $2 \%$ for a transmission of $10^{-4}$.. Instead of the line source that was used for the 1D simulations, a circular surface source was used. The lateral extension of this source $(10 \mathrm{~cm})$ was chosen sufficiently large to make sure that both the collimator and the tally surface (diameter $5 \mathrm{~cm}$ ) were fully covered by the $\gamma$-beam for all relevant angles of incidence $\phi$.

Table 2. MCNP Input parameters for cylindrical collimators.

\begin{tabular}{|c|c|}
\hline Material & Tungsten \\
\hline$D(\mathrm{~cm})$ & 7.0 \\
\hline$S(\mathrm{~cm})$ & 0.0 \\
\hline $\tan \alpha$ & 0.1 \\
\hline $\mathrm{nps}$ & $10^{8}$ \\
\hline
\end{tabular}

The results for cylindrical collimators are shown in Figures 14 and 15. From these figures it can be observed that the transmission of a beam of parallel $\gamma$-rays behaves in a very similar way as for one dimensional collimators. For the DKE case the transmission curves show a curved profile within the acceptance range, whereas for SKE collimators the transmission profile is practically flat.

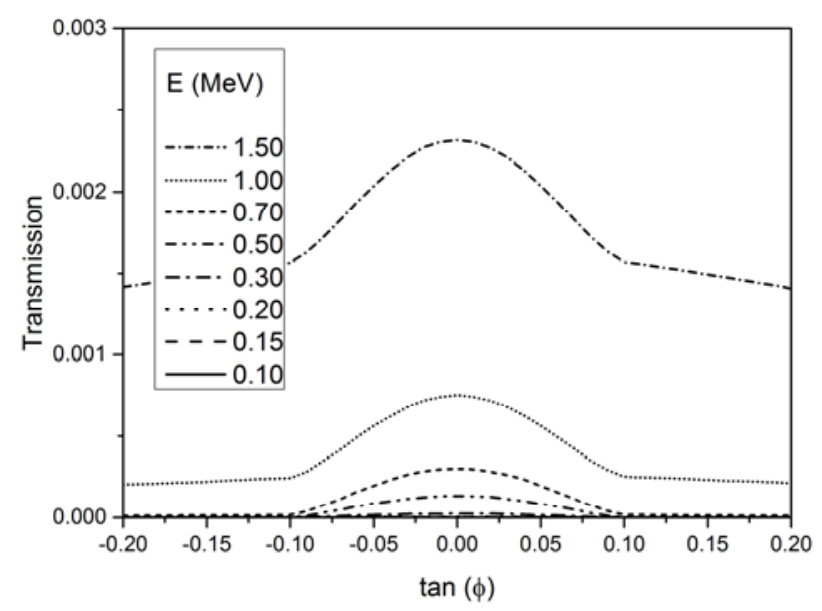

Fig. 14. Transmission curves for cylindrical DKE collimators for various $\gamma$-energies. The other relevant parameters are given in Table 2.



Fig. 15. Transmission curves for cylindrical SKE collimators for various $\gamma$-energies. The other relevant parameters are given in Table 2.

\subsection{Reversed cylindrical collimators}

Because reversed cylindrical SKE collimators are occasionally used in imaging studies [5], these were also investigated. In a reversed SKE collimator, the conical opening diverges towards the detector. The results are shown in Figure 16. Apart from small differences due to the statistical nature of the simulations, the results shown in Figure 16 are identical with those from Figure 15.

This result shows that for this particular case (zero split and parallel $\gamma$-beam) the forward $v s$. backward orientation of the collimator does not influence its transmission characteristics.

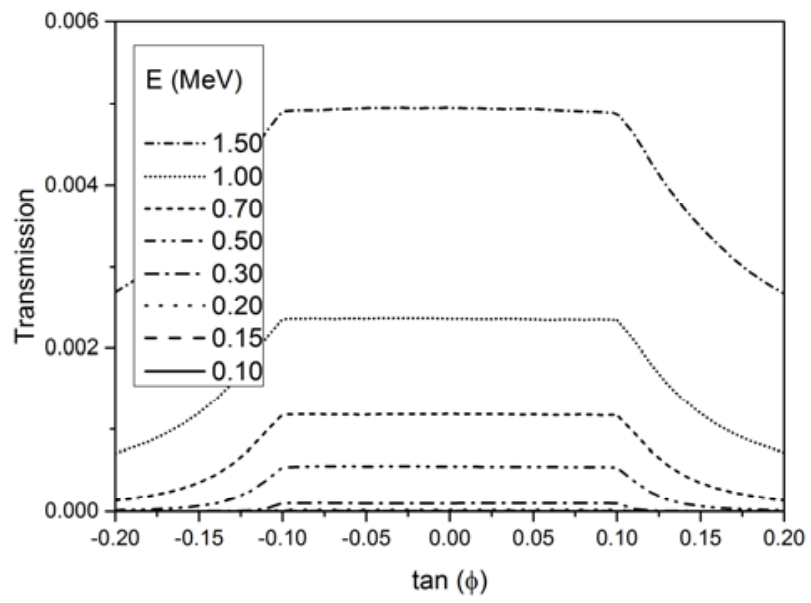

Fig. 16. Transmission curves for reversed cylindrical SKE collimators for various $\gamma$-energies. The other relevant parameters are given in Table 2. 


\section{Discussion}

\subsection{Comparison with theoretical results}

\subsubsection{Cylindrical DKE collimator}

In Figure 17 we show a comparison between the results from our simulation, and the theoretical formula by Metzler et al. [6]. The constant $C$ in formula 1 was adapted to match the maxima of both curves. The parameters used for the simulation are as in Table 2, and the $\gamma$-energy was set to $0.5 \mathrm{MeV}$.



Fig. 17. Comparison between MCNP simulations for a 3D DKE collimator and theoretical results by Metzler et al. [6].

From this figure it can be seen that there is a very good agreement between theory and simulation. Note, however, that for higher $\gamma$-energies the simulations predict a distinct tail for angles of incidence outside acceptance angle (as shown e.g. in Fig. 14), whereas Equation (1) predicts zero transmission for these conditions.

\subsubsection{One dimensional DKE collimator}

In Figure 19 we show a comparison between the results from our simulation and the theoretical formula by Metzler et al. [6]. The constant $C$ in formula 1 was adapted to match the maxima of both curves. The parameters used for the simulation are as in Table 2, and the $\gamma$-energy was set to $0.5 \mathrm{MeV}$.

From Figure 19 it can be seen that the theoretical formula describes a somewhat narrower profile than the simulations. However, the theoretical formula that was derived for a 3D cylindrical collimator and therefore does not necessarily apply to the $1 \mathrm{D}$ case for which the simulations were carried out.



Fig. 18. Comparison between MCNP simulations for a 1D DKE collimator and theoretical results by Metzler et al. [6] for a $3 \mathrm{D}$ cylindrical collimator.

\subsubsection{Cylindrical SKE collimator}

In Figure 18 we show a comparison between the results from our simulation, and the theoretical formula by Alnaghy et al. [7]. The constant $C$ in formula 2 was adapted to match the maxima of both curves. The parameters used for the simulation are as in Table 2, and the $\gamma$-energy was set to $0.5 \mathrm{MeV}$.

From this figure it can be seen that there is little agreement between theory and simulation. The reason for this discrepancy is unclear, but is must be noted that the theoretical formula is an approximation for the case of a point source, located closely to the collimator, whereas the simulations were carried out for a source at infinite distance.

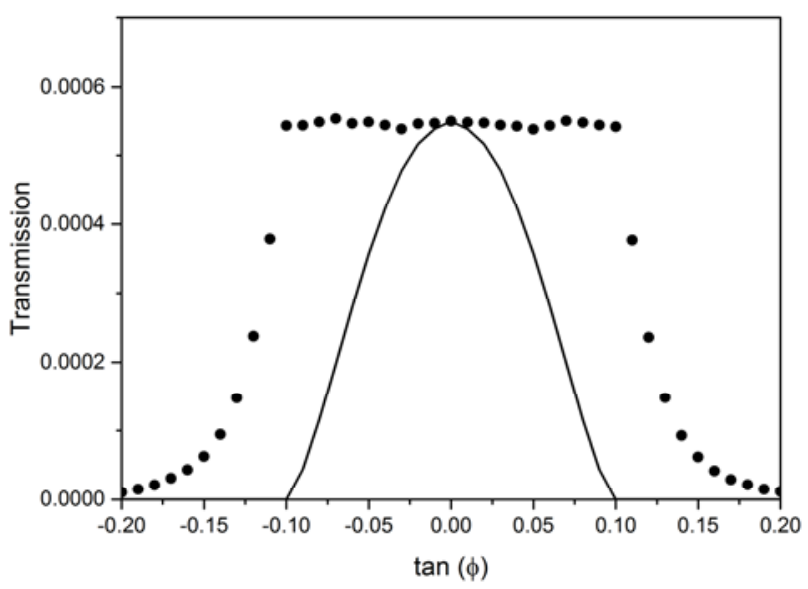

Fig. 19. Comparison between MCNP simulations and theoretical results by Alnaghy et al. [7]. 


\subsection{Qualitative explanation}

Since there is a marked discrepancy between the theoretical formula by Alnaghy et al. [7] and the MCNP simulations reported in this paper, a simple geometrical argument will be presented, that qualitatively explains the observed behaviour of the different collimator types. It is assumed that $\gamma$-rays, which have to transverse less than half the thickness $D$ of the collimator, pass without attenuation while $\gamma$-rays, that have to transverse a greater length, are completely absorbed. This is illustrated in Figure 20 for the DKE collimator.

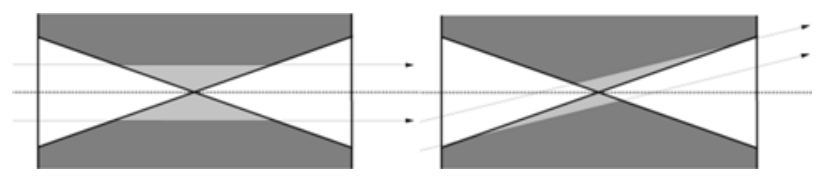

Fig. 20. Qualitative explanation of the angular dependence of the transmission of a DKE collimator. The transparent region is coloured light grey, the opaque region dark grey.

From this figure it can be observed that the effective open area of the collimator rapidly diminishes with increasing angle of incidence, and will completely be closed when the incoming beam grazes the collimator surface, i.e. when $\phi$ equals $\alpha$. The case of the SKE collimator is illustrated in Figure 21.


Fig. 21. Qualitative explanation of the angular dependence of the transmission of a SKE collimator. The transparent region is coloured light grey, the opaque region dark grey.

From this figure it can be observed that the effective open area of the collimator does not change with increasing angle of incidence. Beyond grazing incidence, i.e. when $\phi>\alpha$, the transmitted fraction of the beam will be gradually reduced. This qualitative model describes the results from the MCNP simulations quite well.

\section{Conclusions}

In this paper we have reported results on MCNP calculations used to determine the optimal type of collimator to be used for vertical collimation in a waste scanning system. From the two collimator types that were studied, the SKE collimator was found to have a transmission curve that is practically independent of the angle of incidence, whereas the DKE collimator shows strong variations in transmission. Therefore the SKE collimator is to be preferred when a conservative estimate of the activity inside the waste vessels has to be reported.

A disadvantage of the SKE collimator is that it shows considerable flux leakage outside of the angle of acceptance, especially for high $\gamma$-energies. This can be remedied to some extent by increasing the thickness of the collimator.

For waste vessels of lower activity the transmission can be increased by increasing the width of the collimator split. In that case the flat transmission profile of the SKE collimator is preserved, but at larger split widths the difference between SKE and DKE collimators gradually disappears.

Theoretical results for knife edge collimators are only available for cylindrical collimators. They agree well with our MCNP results for DKE collimators, but for SKE collimators marked discrepancies were found.

Further theoretical work on SKE collimators is required to clarify these discrepancies.

\section{Acknowledgements}

We would like to thank Pieter van Mullekom and Jürgen Willems at Nuclear Fields, for providing insight into possible applications of collimating systems, and Marlies Goorden at Delft University for fruitful discussions about the use of collimators in medical imaging. This work was supported by the Dutch Ministry for Economic Affairs. 


\section{References}

1. J Steven Hansen, Application Guide To Tomographic Gamma Scanning Of Uranium And Plutonium, LA-UR-04-7014, Los Alamos National Laboratory (2004) TGS

2. T. Bücherl, E. Kaciniel, Ch. Lierse, Synopsis of Gamma scanning systems, Report WG-A-01, European Commission (1998)

3. https://www.nrg.eu/about-nrg/radioactive-wasteproject/technical-equipment/gamma-scanner-forcharacterisation-vinish.html? L=1
4. D.B. Pelowitz (ed.), MCNPX user's manual, version 2.6.0, Report LA-CP-07-1473, Los Alamos National Laboratory (2008)

5. Oleksandra Ivashchenko, Frans van der Have, Marlies C. Goorden, RuudM. Ramakers, and Freek J. Beekman, J. Nucl. Med. 56, 470 (2015)

6. S.D. Metzler, J.E. Bowsher, M.F. Smith, R.J/ Jaszczak, IEEE Trans. Med. Imaging, 20, 730 (2001)

7. Saree Alnaghy, Mitra Safavi-Naeini, Daniel R. Franklin, Zhangbo Han, Dean L. Cutajar, Marco Petasecca, Michael Lerch, Anatoly B. Rosenfeld, IEEE Trans. Nucl. Sci., 63, 1375 (2016) 